\title{
Interférences
}

Ars scribendi

$9 \mid 2016$

Varia

\section{La retractatio du Peristephanon 10 de Prudence par Flodoard à travers l'intertextualité épique}

Julie Leyronas

\section{(2) OpenEdition}

Journals

Édition électronique

URL : http://journals.openedition.org/interferences/5677

DOI : 10.4000/interferences.5677

ISSN : $1777-5485$

Éditeur

HiSoMA - Histoire et sources des Mondes antiques

Référence électronique

Julie Leyronas, «La retractatio du Peristephanon 10 de Prudence par Flodoard à travers l'intertextualité épique », Interférences [En ligne], 9 | 2016, mis en ligne le 18 janvier 2018, consulté le 15 septembre 2020. URL : http://journals.openedition.org/interferences/5677 ; DOI : https://doi.org/10.4000/ interferences.5677

Ce document a été généré automatiquement le 15 septembre 2020.

Tous droits réservés 


\section{La retractatio du Peristephanon 10 de Prudence par Flodoard à travers l'intertextualité épique}

Julie Leyronas

\section{Texte}

\section{Texte latin ${ }^{1}$}

De triumphis Christi Antiochiae gestis

CAPVT XIV : De sancto Romano

\begin{tabular}{|l|l|}
\hline Romani luctam aureolo Prudentius ore & \\
\hline Intonuit ; quare satis est libare tropaea. & 765 \\
\hline Denique praefectus huc mittitur Asclepiades & \\
\hline Ecclesiamque dolo meditans irrumpere furuo, & \\
\hline Mansuetum Christi disperdere ouile uolutat. & \\
\hline Panditur id sancto superi charismate Regis & 770 \\
\hline Romano, monachi qui relligione decenter & \\
\hline Praepollens, ualido reliquos fulcimine munit & \\
\hline Vt stent exhortans contra dicantque tyranno: & \\
\hline Vnde sibi sisti iubet hunc praefectus ut audit. & 775 \\
\hline Sistitur, arguitur, reperitur fortis, agone & \\
\hline Intrepidus, constans, Domini subnixus amore: & \\
\hline Concutitur flagris, tormento appensus equino ; & \\
\hline
\end{tabular}




\begin{tabular}{|c|c|}
\hline \multicolumn{2}{|l|}{ Laetior efficitur, tortus tormenta triumphans. } \\
\hline Exprobrat excrucians tortorem, uerbera nescit ; & \\
\hline Tristibus ac laetis Christi praeponit amorem & \\
\hline Atque Dei seruos meliores regibus effert. & 780 \\
\hline Depositus quatitur librati uerbere plumbi. & \\
\hline Hinc lectus spondetur honos, ille omnia temnit & \\
\hline Et saecli decus omne Dei postponit honori. & \\
\hline Caeditur hinc lignis, tensus lictore quaterno ; & \\
\hline Tum quoque maxillas subacuta nouacula rasit, & 785 \\
\hline Facundi uocem dolor ut compesceret oris. & \\
\hline Confessor nulla substernitur arte repressus ; & \\
\hline Promptius alloquitur, puerumque admittier orat & \\
\hline Quemlibet, ac uera de relligione rogari & \\
\hline Vnum siue Deum plures dicatue colendos. & 790 \\
\hline Raptatur puer, ostendi martyr petit alti & \\
\hline Virtutem Christi, puerum sic uera reposcens, & \\
\hline De cultu summo absque dolo iubet apta fateri. & \\
\hline Ilicet effatur solum puer esse colendum & \\
\hline Aetheris atque soli Dominum, non scultile uanum. & 795 \\
\hline Angitur hoc haerens stupidus sermone tyrannus ; & \\
\hline Versus in infantem quis ad haec instruxerit illum & \\
\hline Scitatur. Matrem se pusio fatur habere & \\
\hline Christicolam, doctumque ab ea se credere Christo. & \\
\hline Mittitur ascitum matrem ; perducitur illa: & 800 \\
\hline Praecipitur geniti genitrix tormenta tueri. & \\
\hline Confessus se Christicolam suspenditur ille & \\
\hline Torqueturque, pio mater quem robore firmat & \\
\hline Aeterni et fontis sitienti pocula suffit. & \\
\hline Cum puero Barula flagris Romanus adactus, & 805 \\
\hline Miles cum tyrone, nouus cum milite tyro ; & \\
\hline Claudunturque simul torti imo carceris antro. & \\
\hline Interea coluber diras excogitat artes & \\
\hline Lignorumque pyram iubet extra moenia poni. & \\
\hline Fulgebat posthac tenebris lux altera pulsis & 810 \\
\hline Praefectusque ibat populo comitatus opimo ; & \\
\hline
\end{tabular}




\begin{tabular}{|c|c|}
\hline \multicolumn{2}{|l|}{ Ibant et sancti gaudentes igne probari } \\
\hline Ingentemque rogum Romanus feruere cernens & \\
\hline Inuocat excelsum lymphaeque ignisque potentem. & \\
\hline Ecce repente nitens obscurant nubila caelum, & 815 \\
\hline Nubibus et densis tranquillus cogitur aer & \\
\hline Demissusque polo ueniens largissimus imber & \\
\hline Imbuit extinxitque pyram, feruoris ademit & \\
\hline Vim totam; nihil hic flammae, nihil ignis agebat. & \\
\hline Glorificant Christum populi, ferus uritur hostis, & 820 \\
\hline Deficit atque iterum sanctos in carcere trudit. & \\
\hline Hinc iterum spargit cum iam lux crastina terras, & \\
\hline Pelluntur, deducuntur trucis ante tribunal & \\
\hline Iudicis. Ille dolens non se uicisse per ignes, & \\
\hline Succensusque iubet Stygia face et igne Cocyti, & 825 \\
\hline Mox puero tenera caput a ceruice recidi & \\
\hline Romanoque Deum laudans plectrum ore reuelli. & \\
\hline Tum gnati genitrix exultans membra receptat. & \\
\hline Ducitur hinc medicus Romani abscidere uerba, & \\
\hline Accipit et regimen radicitus amputat oris. & 830 \\
\hline Ille gemens caelumque tuens benedicere certat & \\
\hline Clara uoce Deum, sibi qui uocem sine lingua, & \\
\hline Sermonem sine more dedit, sine uerbere uerba. & \\
\hline Praefectus medico tormenta inferre minatur & \\
\hline Quod decus inuicti parcens non dempserit oris. Ille palam monstrat toto abscidisse palato & 835 \\
\hline Plectrum oris, testans aliud non uiuere posse & \\
\hline Sic abscisum animal. Iubet ille adducere porcum, & \\
\hline Cui parili ut secuit chirurgus acumine linguam, & \\
\hline Labitur et pariter cum lingua commoritur sus. & 840 \\
\hline Euadit medicus. Praefectus Maximiano & \\
\hline Nuntiat haec. Ille occulte sub carcere clausum & \\
\hline Romanum iubet occidi ; fit iussio saeui & \\
\hline Principis, et martyr celebrem consummat agonem, & \\
\hline Aeternamque capit caelorum a rege coronam. & 845 \\
\hline
\end{tabular}




\section{Traduction}

\section{Chapitre XIV : Saint Romain}

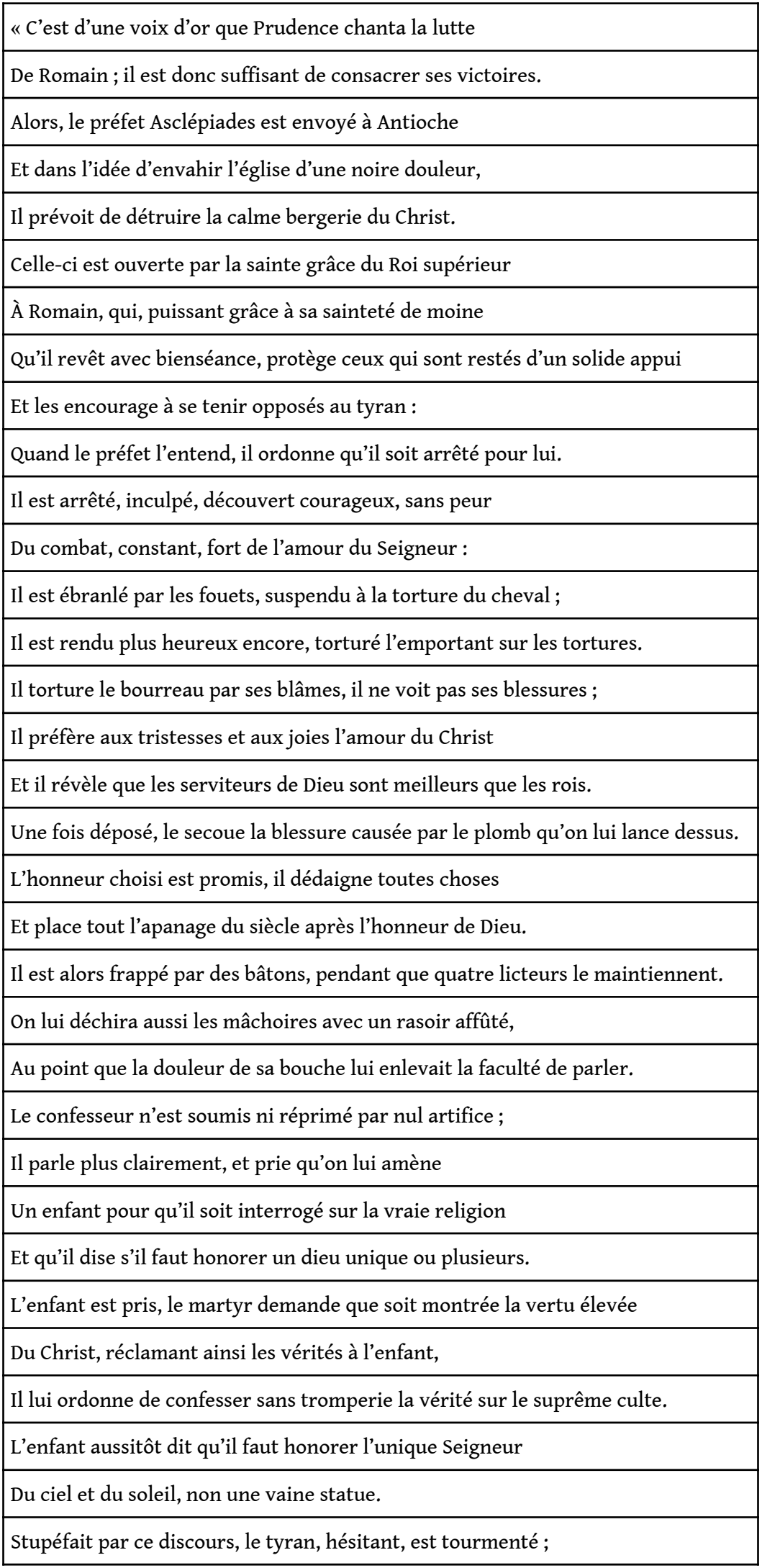




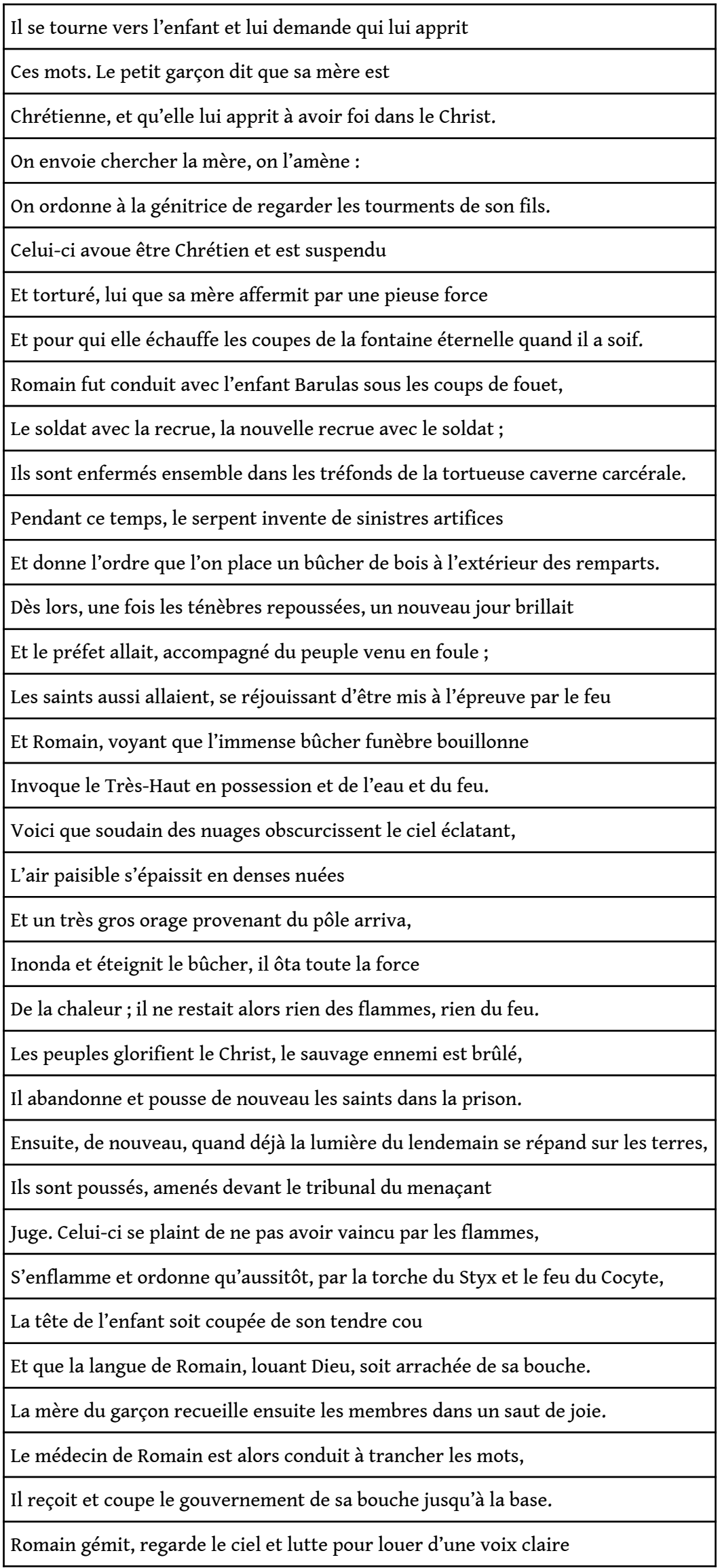




\begin{tabular}{|l|}
\hline Dieu qui lui donna une voix sans langue, \\
\hline Un discours sans désir, des paroles sans blessure. \\
\hline Le préfet menace de torturer le médecin \\
\hline Pour l'avoir préservé et ne pas avoir enlevé la dignité de sa bouche invaincue. \\
\hline Le médecin montre clairement qu'il a coupé la langue de sa bouche \\
\hline Dans tout son palais, témoignant que nul autre animal ne peut vivre \\
\hline Ainsi mutilé. Il ordonne qu'on amène un porc: \\
\hline Lorsque le chirurgien lui coupa la langue avec la même pointe, \\
\hline Le cochon tombe et meurt en même temps que sa langue. \\
\hline Le médecin se sauve. Le préfet informe Maximien \\
\hline Des faits. Celui-ci ordonne que Romain soit enfermé en cachette dans la prison \\
\hline Et tué ; le commandement du cruel empereur \\
\hline Fut accompli, et le martyr achève son illustre combat \\
\hline Et reçoit du roi des cieux la couronne éternelle. » \\
\hline
\end{tabular}

\section{Étude}

1 Le chapitre 14 du livre 1 du De Triumphis Christi Antiochiae gestis de Flodoard est consacré à Romain, diacre de l'Église de Césarée à Antioche, martyr sous la persécution de Dioclétien en $303^{2}$. Ce récit de martyre chez Flodoard fait figure de cas particulier à plus d'un titre. Il s'agit du seul où l'auteur s'inscrit explicitement dans la réécriture d'un auteur ${ }^{3}$, en l'occurrence du Peristephanon $10^{4}$ de Prudence. Par réécriture, nous entendrons la définition donnée par Monique Goullet, à savoir «la rédaction d'une nouvelle version (hypertexte) d'un texte préexistant (hypotexte), obtenue par des modifications appelées formelles pour autant qu'elles affectent le signifiant [...], et sémantiques (ou conceptuelles) pour autant qu'elles affectent le signifié ${ }^{5} »$. Dans ce cas, il s'agit surtout d'une modification affectant le signifiant. De fait, Flodoard réécrit en hexamètres dactyliques un hypotexte déjà en vers. Il faut savoir que les principales sources du De Triumphis Christi Antiochiae gestis sont l'Historia Ecclesiastica d'Eusèbe de Césarée traduite et complétée par Rufin, l'Historia Ecclesiastica Tripartita de Cassiodore, le Martyrologe d'Adon, la Vita Malchi de Jérôme et enfin le De Gloria martyrum et le De Gloria confessorum de Grégoire de Tours ${ }^{6}$. Ces œuvres sont les sources de Flodoard pour 25 des 28 chapitres du De Triumphis Christi Antiochiae gestis ${ }^{7}$. Autrement dit, ce sont des hypotextes en prose que Flodoard transpose en vers. On sait que le passage de la prose aux vers dans l'hagiographie médiévale est une pratique courante, motivée notamment par la volonté de "réécriture en meilleur style ${ }^{8}$ ", pour reprendre l'expression de Monique Goullet. Ainsi, «la réécriture en vers est toujours perçue non seulement comme un acte légitime, mais même comme un enrichissement du texte ${ }^{9}$ ». Dans le cas du martyre de Romain, la source est déjà en vers, mais en mètre iambique, alors que Flodoard compose en hexamètres dactyliques. Nous pourrons donc nous demander quel est l'enjeu d'une telle transposition, d'autant plus que sur les 2490 vers qui composent le De Triumphis Christi Antiochiae gestis, on n'en compte pas moins de 552 
en sénaires iambiques. Mais ici, Flodoard a fait le choix de l'hexamètre dactylique. Ce poème se pose donc comme un simple résumé de la grande œuvre de Prudence mais opère finalement une fusion entre la littérature antique païenne et les thèmes chrétiens grâce à une forme de dialogue entre les auteurs qui s'enrichissent mutuellement. Pour étudier ce phénomène, nous présenterons le projet de Flodoard dans ce poème consacré à Romain pour voir comment sa retractatio opère un changement de genre littéraire et fait du martyre de Romain une épopée en miniature. Enfin, nous mettrons en lumière le dialogisme entre les auteurs païens et les valeurs chrétiennes que permet Flodoard.

2 Commençons par analyser la posture énonciative de Flodoard au début de son poème et la présentation de son projet d'écriture. L'hymne 10 du Peristephanon de Prudence débute sur une inuocatio, non adressée à la Muse, mais à Romain, qui doit inspirer ce chant à Prudence, alors même que ce martyr a été privé de sa langue. Mais justement, comme l'écrit Prudence au vers 22, sa langue sera le Christ (mea lingua Christi). Cette captatio beneuolentiae lui permet de célébrer la grandeur de Dieu. L'enjeu est donc de taille pour qui veut écrire sur Romain après Prudence. Si Flodoard revendique sa source, il commence son poème sur la posture éthique topique du poète en tant que successeur inférieur. C'est pourquoi il annonce dès le vers 765 qu'il ne développera pas le récit du martyre de Romain :

\begin{tabular}{|l|l|}
\hline Romani luctam aureolo Prudentius ore & \\
\hline Intonuit ; quare satis est libare tropaea. & 765 \\
\hline & \\
\hline «C'est d'une voix d'or que Prudence chanta la lutte & \\
\hline De Romain; il est donc suffisant de consacrer sa victoire. » (nous traduisons $\left.{ }^{\text {a }}\right)$ & \\
\hline a. Toutes les traductions de Flodoard que nous donnerons sont personnelles. \\
\hline
\end{tabular}

3 Le vers 765 est fort intéressant par sa structure métrique. Il peut accepter les trois coupes : trihémimère, penthémimère et hephthémimère. La première isole le verbe intonuit, louant ainsi Prudence. Si l'on opte pour une coupe penthémimère, cette fois-ci c'est l'adverbe quare qui est mis en avant, soulignant ainsi le lien entre Prudence et Flodoard, et surtout la captatio beneuolentiae par laquelle le poète se pose en écrivain inférieur à sa source. Mais si l'on garde la coupe trihémimère, doublée de l'hephtémimère, cette dernière met en exergue le groupe satis est qui, tout en ressortissant toujours de la captatio beneuolentiae, a une valeur programmatique: Flodoard fera un récit bien plus court que celui de Prudence. Or la breuitas est aussi un topos de la réécriture hagiographique ${ }^{10}$. Dans notre cas, c'est un fait indéniable: l'hymne 10 du Peristephanon ne compte pas moins de 1140 vers, que Flodoard réécrit en 81 vers. D'ailleurs, la Bibliotheca Hagiographica Latina ${ }^{11}$ présente le récit du martyre de Romain chez Prudence comme étant un carmen, alors qu'elle mentionne les epitomae de Flodoard. Pour en revenir à ce dernier, la coupe hephtémimère du vers 765 isole et met en valeur aussi le dernier groupe du vers, libare tropaea. Or, ce dernier élément, de façon implicite, présente aussi le projet de Flodoard. En effet, les termes libare et tropaea ne sont pas présents chez Prudence (et comme nous aurons l'occasion de le constater, notre auteur n'hésite pas à reprendre des mots précis présents dans l'hypotexte), mais 
on peut voir là une réminiscence virgilienne. Dans l'Énéide, le nom tropaeum est fréquemment placé en fin de vers $(A .10,542 ; 10,775 ; 11,7 ; 11,224 ; 11,385 ; 11,790)$. On remarque que les occurrences virgiliennes se trouvent toutes dans les livres qui décrivent les combats entre Énée et Turnus, autrement dit dans ceux où la dimension épique est la plus prégnante. Or, en faisant le choix de transposer du mètre iambique à l'hexamètre dactylique le récit du martyre de Romain, Flodoard opte pour un ancrage héroïque de cette histoire, bien plus que Prudence. Nous allons étudier comment, grâce à ce développement de la dimension épique, Flodoard fait dialoguer le christianisme à travers Prudence et les auteurs païens latins. Autrement dit, malgré l'annonce préliminaire de breuitas dans sa retractatio, le poème de Flodoard en dirait davantage qu'il n'y paraît et l'enjeu de cette réécriture se situerait justement dans l'utilisation des réminiscences qui ne sont plus ici de simples chevilles métriques ${ }^{12}$, si l'on peut dire, permettant de débuter ou terminer un hexamètre dactylique.

Flodoard procède à un changement de mètre et, par conséquent, à un changement de genre littéraire. Partons du projet de Prudence dans le Peristephanon 10. L'utilisation de l'iambe est caractéristique du mètre parlé dans la tragédie, par sa proximité avec le rythme naturel de la langue de tous les jours. On note une grande place accordée au discours direct chez Prudence: près des deux tiers des 1140 vers qui forment le Peristephanon constituent du discours direct. Or, on constate l'absence totale de discours direct chez Flodoard. Cela peut constituer une première justification du changement de mètre. Mais cette explication demeure insuffisante, si ce n'est irrecevable, au vu du reste du De Triumphis Christi Antiochiae gestis. Ainsi le chapitre 7 du livre 1, consacré à Maximin et Sérapion, est bien composé en sénaires iambiques mais ne contient pas plus de discours direct que le récit du martyre de Romain. On sait que les mètres iambiques sont très employés au théâtre, puisqu'ils peuvent «se plier à toutes les nuances de la conversation ${ }^{13} »$. Or, l'hymne $10 \mathrm{du}$ Peristephanon a pu être comparé à une tragédie. Pour Laurence Gosserez, ce " poème est comparable à une tragédie non seulement par sa longueur exceptionnelle (1 140 vers), mais par son mètre, le trimètre iambique ${ }^{14}$ ». On retrouve des thèmes présentés par Aristote comme représentatifs de la tragédie : la reconnaissance et les péripéties ${ }^{15}$. Dans le cas de l'histoire de Romain, l'objet de la reconnaissance n'est autre que l'existence de Dieu. Et au vers 1113, Prudence précise que le préfet qui juge Romain «relatait méthodiquement tout le développement de cette longue tragédie » : seriemque tantae digerens tragoediae. Qui plus est, la version de Prudence possède des caractéristiques qui correspondent aux règles de la tragédie classique : le personnage principal, véritable "héros tragique ${ }^{16}$ ", est tué hors scène (dans le cachot et non sur la place publique où il est torturé) et les trois unités sont respectées. Ainsi, la tragédie de Prudence se déroule en un lieu, a pour objet le martyre de saint Romain et le tout se déroule sur une seule journée ${ }^{17}$. Or, si Flodoard respecte assez scrupuleusement sa source, on remarque qu'il n'en est rien en ce qui concerne l'unité de temps. Chez Flodoard, après l'échec de la mort par le bûcher, le poète écrit au vers 822 : Hinc iterum spargit cum iam lux crastina terras, "Alors, quand de nouveau la lumière du lendemain se répand sur les terres ». L'adverbe iterum peut être entendu dans le sens large de «à nouveau ", mais aussi dans son sens premier, " pour la seconde fois ». Et cette interprétation éclairerait la lecture du vers 810. Pour faciliter la compréhension, nous citons le texte des vers 808 à 811 , juste après que Romain et l'enfant Barulas ont été enfermés dans un cachot: 


\begin{tabular}{|l|l|}
\hline Interea coluber diras excogitat artes & \\
\hline Lignorumque pyram iubet extra moenia poni. & \\
\hline Fulgebat posthac tenebris lux altera pulsis & 810 \\
\hline Praefectusque ibat populo comitatus opimo & \\
\hline & \\
\hline « Pendant ce temps, le serpent invente de sinistres artifices & \\
\hline Et donne l'ordre que l'on place un bûcher de bois à l'extérieur des remparts. & \\
\hline Dès lors, une fois les ténèbres repoussées, un nouveau jour brillait & \\
\hline Et le préfet allait, accompagné du peuple venu en foule. » & \\
\hline
\end{tabular}

5 On pourrait comprendre que l'ordre donné par le préfet Asclépiades de dresser un bûcher est immédiatement suivi de son exécution, comme c'est le cas chez Prudence (v. 811-817). Mais ce n'est absolument pas clair chez Flodoard. De plus, les ténèbres peuvent aussi bien désigner le démon (et dans ce cas, ce vers préfigure l'échec du bûcher) que les ténèbres de la nuit. Mais la présence de l'adverbe iterum laisse penser qu'il s'agit de la seconde fois que le jour se lève depuis le début du martyre de Romain. En insistant en si peu de vers sur le déroulement des jours et des nuits, qui plus est par deux fois, Flodoard exprime implicitement sa volonté de se démarquer de son hypotexte et de s'ancrer dans le genre épique. Il s'écarte ainsi de l'unité de temps et donc de la dimension théâtrale si prégnante chez Prudence. Outre l'hexamètre dactylique, une des marques de l'épopée se trouve dans les «transitions temporelles qui évoquent l'alternance du jour et de la nuit qui donne une emphase épique au récit ${ }^{18}$ ». Or, il est intéressant de constater que, dans ces deux vers (v. 810 et 822), qui ne correspondent à aucun vers de Prudence, foisonnent les réminiscences épiques. Le vers 810 s'ouvre sur le verbe fulgebat. Il s'agit d'un début d'hexamètre classique que l'on retrouve en particulier chez Virgile $(A .11,490)$ et Silius Italicus $(7,444)$. On peut préciser que l'occurrence de fulgebat en début de vers chez Virgile se situe à un moment de combat: ce verbe s'applique à Turnus qui étincelle d'or. On se trouve dans une référence à un passage épique essentiel de l'Énéide: le combat entre Énée et Turnus. Toujours concernant le vers 810 de Flodoard, on observe une autre réminiscence cette fois-ci dans l'image des ténèbres chassées. On pense ici aux Métamorphoses d'Ovide ( 7 , 703) : lutea mane uidet pulsis Aurora tenebris, «l'Aurore, dont la lumière de safran venait de chasser les ténèbres ${ }^{19} \rrbracket$. Même si les éléments de l'ablatif absolu ne sont pas situés à la même place métrique chez Ovide et chez Flodoard, on constate que, dans les vers des deux auteurs, une coupe penthémimère isole le nom et le participe qui constituent le cœur de l'ablatif absolu et encadrent l'élément de lumière. Et dans les deux cas, le mot qui désigne la lumière (Aurora chez Ovide, lux chez Flodoard) jouxte l'adjectif tenebris. Ce fait peut expliquer l'inversion des mots tenebris et pulsis chez Flodoard. Le travail opéré sur cette partie du vers suggère une réminiscence volontaire de la part de l'auteur médiéval. Là encore, Flodoard emprunte à un poème épique, celui qui raconte les origines du monde et de la grandeur de Rome... Le vers 822 , quant à lui, emprunte ses premiers mots à l'épopée de Silius Italicus $(11,536)$ : hinc iterum repetens restat nunc sedibus imis. De plus, l'expression lux crastina se retrouve à cette même place également chez Silius Italicus $(14,669)$ et chez Stace $($ Theb. 10, 21). Ces deux vers nous montrent 
donc le recul que prend Flodoard vis-à-vis de sa source Prudence afin d'accentuer le registre épique de son poème grâce au détournement de la temporalité.

Ce jeu sur la temporalité passe aussi par une modification du déroulement des faits : Flodoard s'écarte de la chronologie de Prudence dans l'enchaînement des événements. Chez Prudence, Asclépiades annonce dès le vers 825 , juste après les paroles de l'enfant et ses premières tortures, qu'il fera périr Romain et Barulas dans des morts différentes, le premier sur le bûcher, le second par la décapitation. Et l'enfant est aussitôt décapité (v. 831-845). La description du bûcher, réservé uniquement à Romain, commence au vers 846. Chez Flodoard, Barulas mourra également décapité (v. 826). Mais avant cela, il était destiné lui aussi au bûcher. C'est l'orage qui lui permet d'échapper au supplice par le feu. Dans le Martyrologe d'Adon, on trouve le même déroulement des faits que chez Prudence. Comment expliquer cette modification chez Flodoard? On peut voir là une manière supplémentaire de mettre en valeur l'épisode du bûcher et surtout la dimension épique du poème de Flodoard. Elle est en effet intensifiée si Romain ne se dirige pas seul vers le bûcher. Cette idée se rapproche de ce que Paul-Augustin Deproost nomme le «compagnonnage hérö̈que ${ }^{20} »$. Sur les modèles homérique et virgilien, le héros n'est pas seul mais accompagné de personnes qui partagent ses valeurs. Cela permet de mettre en valeur le personnage principal comme héros épique capable de rassembler par ses vertus héroïques. Cela ressort d'ailleurs du fait que l'on trouve le même début de vers (ibant et) chez Virgile $(A .9,369)$ pour désigner l'armée qui s'avance. Cette union de compagnons qui partagent des valeurs est d'autant plus frappante chez Flodoard qu'au vers précédent (v. 811$)$ le verbe ire est déjà employé à l'imparfait, mais au singulier : Asclépiades ibat. Il est certes accompagné du peuple venu en foule (ibat populo comitatus opimo). Mais cet accompagnement ne marque pas une communauté de valeurs, bien au contraire. C'est pourquoi, contrairement au vers suivant, l'union n'est pas marquée par un seul verbe au pluriel.

7 Cette première caractéristique du héros accompagné d'un fidèle comparse se double d'une autre qualité, bien propre au héros épique, que Flodoard fait une nouvelle fois ressortir grâce à l'intertextualité. Le vers 775 s'ouvre sur l'adjectif intrepidus qui révèle une particularité essentielle du héros épique : il est dénué de peur. Or, cet adjectif, qui plus est employé à cette place, appelle une foule de références épiques. On le trouve en effet chez Silius Italicus $(4,460$ : Intrepidus puer et Gradiuum passibus aequat) : en plein champ de bataille, un jeune homme veut venger son père. Il est rendu invincible par la protection divine invisible de Gradivus/Mars, envoyé par Jupiter. De même, chez Flodoard, Romain est rendu sans peur grâce à l'action divine au moment de son arrestation. Bien sûr, l'absence de peur constitue une valeur héroïque essentielle. Qui plus est, la présence de cet adjectif en début d'hexamètre dactylique correspond à une place courante (Stace, Theb. 11, 687 ; Lucain, 2, 207 ; 5, $658 ; 6,219$ ).

Nous pouvons relever encore une caractéristique du genre épique que l'on trouve chez Flodoard et qu'il peut développer justement grâce aux réminiscences: les mots de transition et autres expressions formulaires ${ }^{21}$. La volonté de breuitas exprimée par Flodoard est bien présente. Ainsi, le vers 785 débute sur la formule tum quoque qui permet à l'auteur de passer sous silence les paroles de Romain lorsqu'il est torturé, suspendu sur le chevalet, paroles qui n'occupent pas moins de 86 vers chez Prudence (v. 459-545). Mais cet abrègement passe par le recours à une formule de transition fréquente à cette place chez des auteurs d'épopées, comme Virgile, Ovide, Lucain et Silius Italicus (A. 8, 397; 9, $183 ;$ Met. 1,$527 ; 3,504 ; 4,339 ; 5,56 ; 5,97 ; 5,232 ; 6,18 ; 8$, 
$845 ; 13,571 ; 14,369$; Bell. ciu. 5, $625 ; 9,463$; Pun. 11, 478; 15, 324; 15, 686; 16, 237). Nous pouvons ainsi constater que la réécriture de Flodoard s'effectue sur un mode épique grâce à de multiples références à des épopées latines païennes.

9 Mais quel est l'enjeu de cette retractatio qui tend vers l'épopée? Il est vrai que le Peristephanon 10 comprend déjà de nombreuses réminiscences des auteurs antiques. Ainsi que l'explique Pierre-Yves Fux, "la uariatio est au cœur de la poétique de Prudence $^{22}$ ». Les sources principales de Prudence sont Horace, bien sûr Virgile, mais encore Lucrèce, Properce, Catulle, Ovide, Stace, Juvénal, Sénèque et Lucain ${ }^{23}$. Il n'est donc pas surprenant de retrouver des références à ces auteurs chez Flodoard. Néanmoins, on constate que les réminiscences présentes chez Flodoard ne sont pas celles utilisées par Prudence. Il convient de bien faire le départ entre les formules déjà présentes chez Prudence et que Flodoard réutilise et les réminiscences d'auteurs païens inédites par rapport à Prudence.

Tout d'abord, on trouve chez Flodoard des réminiscences littéraires qui s'opèrent à partir d'un lexique déjà présent chez Prudence. Par exemple, juste après les paroles de l'enfant Barulas qui révèle qu'il n'y a qu'un seul vrai Dieu, le préfet Asclépiades reste stupéfait, ainsi qu'on le lit chez Prudence, vers 676: Stupuit tyrannus sub pudore fluctuans. Or, ce vers a son pendant chez Flodoard, vers 796 : Angitur hoc haerens stupidus sermone tyrannus. Ce vers est clairement une réécriture de Prudence. Outre le sens similaire, on retrouve un écho entre stupuit et stupidus, d'autant plus que ces deux mots sont situés à des places métriques de choix (en début de vers pour stupuit et à la coupe penthémimère pour stupidus). Et dans les deux cas, on retrouve le nom tyrannus au nominatif pour désigner Asclépiades. Mais ce nom en dernière position d'un hexamètre dactylique s'inscrit aussi dans une tradition épique : on le retrouve chez Virgile (A. 10, 448 et 12,75), dans le premier cas, au moment du combat entre Pallas et Turnus, dans le second, lors de l'affrontement final entre Énée et Turnus. Autrement dit, les réminiscences sont toujours puisées dans des temps forts épiques. Prenons un autre exemple. Décrivant l'attitude de la mère de Barulas face au martyre de son enfant, Prudence écrit au vers 715 firmatque sensum mollis indulgentiae, " aux sentiments d'une douce tendresse elle ajoute de la fermeté». Ce passage devient chez Flodoard: Torqueturque, pio mater quem robore firmat, « il est torturé mais sa mère l'affermit par une pieuse force». Encore une fois, on retrouve un terme identique, en l'occurrence le verbe firmat, mis en valeur par sa place dans le vers chez nos deux auteurs. Mais chez Flodoard, ce verbe est complété de l'ablatif robore, au dactyle $5^{\mathrm{e}}$, ce qui est pour ce mot une place courante dans l'épopée latine. Ainsi chez Virgile (A. 6, 214 : Principio pinguem taedis et robore secto ${ }^{24}$ ), mais aussi chez Ovide (Met. 8, 769-770: Detruncat que caput repetita que robora caedit / Editus et medio sonus est de robore talis; 12, 329 : Pectora cum duro luctantia robore fixit). Avec ces quelques exemples, l'emploi de robore dans la position du dactyle $5^{\mathrm{e}}$ pourrait apparaitre comme une simple cheville métrique. Mais l'enjeu héroïque est bien présent à l'aune d'une autre réminiscence épique. On retrouve ainsi dans ce vers une référence à Lucain qui écrit dans le Bellum Ciuile $(2,245): \ldots$ tu mente labantem / derige me, dubium certo tu robore firma ${ }^{25}$, «... dirige mon esprit qui chancelle, dissipe mes doutes et assure moi par ta robuste fermeté ${ }^{26}$. Il s'agit du moment où Brutus va trouver Caton pour lui demander des conseils. Alors que chez Virgile et Ovide robore renvoyait bien au sens premier, au chêne, chez Lucain il faut comprendre le sens abstrait de "force", comme dans le texte de Flodoard. Dans ce passage, Flodoard est bien fidèle au programme annoncé au début de son poème, à savoir un abrègement de sa source (il ne livre pas le contenu des paroles de la mère de 
Barulas, contrairement à Prudence). Dans cet abrègement, il opère bien une retractatio en reprenant les termes mêmes de son hypotexte mais, à partir de ce terme qu'il reprend, il fait dialoguer le texte chrétien de Prudence et les auteurs antiques païens, ce qui lui permet de donner une résonnance épique plus forte au combat mené par Romain. Flodoard ne narre donc pas directement cette lutte, mais il la laisse raconter par d'autres auteurs, établissant ainsi ce que Paul-Augustin Deproost appelle « une étroite collaboration entre antiquité et christianisme ${ }^{27}$ ».

11 Cela pose ici la question du lectorat. Flodoard établit ainsi une connivence avec ses destinataires et fait donc appel à un lectorat cultivé, à une élite intellectuelle ${ }^{28}$. De la même façon, le vers 831 de Flodoard débute sur la réaction de Romain lorsque le médecin lui coupe la langue : Ille gemens... Flodoard reprend le vers 927 de Prudence : gemitu querellam traxit et sic orsus est, «Romain [...] fit entendre un gémissement, une plainte et commença ainsi ». Le participe gemens constitue une retractatio de gemitu, d'autant plus que les deux termes se situent au début du vers. Mais le groupe ille gemens chez Flodoard prend une nouvelle résonnance puisqu'il s'agit d'une réminiscence de Lucain $(5,739$ : Ille gemens non nunc vita mihi dulcior, inquit cum). L'objectif de Flodoard est de faire de Romain un athlète de la foi ${ }^{29}$, un héros épique, grâce à l'intertextualité. Il élabore ainsi « la grande épopée martyriale » dont parle F. Dolbeau ${ }^{30}$.

Mais est-ce à dire que Flodoard subordonne la littérature latine païenne aux thèmes chrétiens ? La retractatio de Flodoard, qui opère un dialogue entre hypotexte chrétien et réminiscences païennes, n'apparaît pas uniquement dans la forme mais aussi dans les thèmes. Ainsi l'épisode du bûcher, que l'on trouve bien sûr chez Prudence, est traité de façon épique par Flodoard. Dans les 81 vers qui composent le chapitre sur saint Romain, les vers 808 à 821 sont consacrés à cet épisode qui, comme nous l'avons dit, est déjà mis en valeur car il se tient sur une seule journée, étant ainsi séparé des autres tortures. Le fait de consacrer 14 vers à un passage précis alors même que Flodoard avait annoncé sa breuitas n'est pas anodin. D'ailleurs, si l'on dénombre 43 réminiscences d'auteurs latins païens sur les 81 vers du chapitre consacré à Romain, 13 d'entre elles sont répertoriées parmi ces 14 vers, ce qui constitue une importante proportion. Nous notons en gras les mots comprenant des réminiscences dans ce passage :

\begin{tabular}{|l|l|}
\hline Interea coluber diras excogitat artes & \\
\hline Lignorumque pyram iubet extra moenia poni. & \\
\hline Fulgebat posthac tenebris lux altera pulsis & 810 \\
\hline Praefectusque ibat populo comitatus opimo ; & \\
\hline Ibant et sancti gaudentes igne probari & \\
\hline Ingentemque rogum Romanus feruere cernens & \\
\hline Inuocat excelsum lymphaeque ignisque potentem. & 815 \\
\hline Ecce repente nitens obscurant nubila caelum, & \\
\hline Nubibus et densis tranquillus cogitur aer & \\
\hline Demissusque polo ueniens largissimus imber & \\
\hline Imbuit extinxitque pyram, feruoris ademit & \\
\hline Vim totam ; nihil hic flammae, nihil ignis agebat. & \\
\hline
\end{tabular}




\begin{tabular}{|l|l|}
\hline Glorificant Christum populi, ferus uritur hostis, & 820 \\
\hline Deficit atque iterum sanctos in carcere trudit. & \\
\hline & \\
\hline « Pendant ce temps, le serpent invente de sinistres artifices & \\
\hline Et donne l'ordre que l'on place un bûcher de bois à l'extérieur des remparts. & \\
\hline Une autre lumière brillait ensuite, une fois les ténèbres repoussées, & \\
\hline Et le préfet allait, accompagné du peuple venu en foule; & \\
\hline Les saints aussi allaient, se réjouissant d'être mis à l'épreuve par le feu & \\
\hline Et Romain, voyant que l'immense bûcher funèbre bouillonne, & \\
\hline Invoque le Très-Haut en possession et de l'eau et du feu. & \\
\hline Voici que soudain des nuages obscurcissent le ciel éclatant & \\
\hline L'air paisible s'épaissit en denses nuées & \\
\hline Et un très gros orage provenant du pôle arriva, & \\
\hline Inonda et éteignit le bûcher, il ôta toute la force & \\
\hline De la chaleur ; il ne restait alors rien des flammes, rien du feu. & \\
\hline Les peuples glorifient le Christ, le sauvage ennemi est brûlé, & \\
\hline Il abandonne et pousse de nouveau les saints dans la prison. » & \\
\hline
\end{tabular}

13 À première vue, cet extrait laisse apparaître des réminiscences qui ne seraient que de simples chevilles métriques de par leur position clef dans le vers (début et fin de vers, dactyle $5^{\mathrm{e}}$ ). Néanmoins, on observe que ce n'est pas le cas aux vers 808 et 810 . Nous avons déjà abordé les réminiscences épiques du vers 810 . Dans le vers 808 , si la place du nom artes en fin de vers est tout à fait classique ${ }^{31}$, l'emploi de ce mot accompagné de l'adjectif diras fait écho à la Guerre punique de Silius Italicus (14, 216 : haud impune quidem ; nam dirae conditor artis). Chez Silius, ce vers se situe au beau milieu du catalogue des alliés de Syracuse (il s'agit de l'histoire du taureau de Phalaris, tyran d'Agrigente), autrement dit dans un épisode épique traditionnel. Or, la réminiscence du vers 816 nous place elle aussi dans un passage épique traditionnel. Le début (nubibus) est bien une réminiscence de Lucrèce $(2,1100 ; 6,125 ; 6,198 ; 6,325 ; 6,496)$. Tous ces passages de Lucrèce analysent le phénomène naturel qu'est la tempête. Mais la fin du vers de Flodoard (cogitur aer) a pour seul écho dans la littérature antique Virgile $(A .5,20)$ : Consurgunt venti, atque in nubem cogitur aer, "Les vents [...] accourent [...], l'air se condense en brouillard». Ce vers prend place au début du livre $5:$ le bateau d'Énée quitte Carthage mais son équipage aperçoit les flammes du bûcher de Didon. Une tempête se lève alors. Or, la tempête constitue un «thème obligé de l'épopée latine ${ }^{32}$ ». La présence d'un bûcher dans les deux textes crée un lien évident entre eux. Mais le lien s'établit aussi dans l'échec de la tempête dans le sens où, dans les deux cas, elle ne porte pas préjudice aux héros. Chez Virgile en effet, l'épisode de la tempête comme obstacle au destin du héros avorte puisque le pilote Palinure propose de ne pas lutter contre elle, mais au contraire de se laisser guider, conseil que suit Énée qui s'écrit (v. 22) : Superat quoniam Fortuna, sequamur, «Puisque la fortune l'emporte, suivons-le ». Et ils sont alors conduits en Sicile, où aura lieu la rencontre avec la Sibylle. De la même 
façon, chez Flodoard, la tempête s'avère être un allié du héros. Il ne s'agit plus alors d'une représentation de la Fortune mais de Dieu. Les phénomènes météorologiques sont des éléments traditionnels pour témoigner de la puissance divine. C'est ce que Michel Sot nomme des visions cosmiques : ainsi, « le ciel est en correspondance avec le monde : des signes visibles y préfigurent les troubles politiques ou biologiques ${ }^{33} »$. Mais ici, cette marque divine prend une ampleur épique avec la résonnance virgilienne. D’ailleurs le vers 815 qui annonce le début de la tempête chez Flodoard est déjà chargé de résonnance épique puisque la fin du vers, nubila caelum, se retrouve chez plusieurs auteurs d'épopées latines païennes, sous la forme nubila caelo ${ }^{34}$. Face à de tels événements, les personnages se comportent en véritables héros. De fait, ibant et sancti gaudentes igne probari, "les saints s'avançaient aussi, se réjouissant d'être mis à l'épreuve par le feu ». Ce début (ibant et) que l'on trouve déjà chez Virgile (A. 9, 369), a été repris par Silius Italicus $(8,420)$ : Ibant et laeti pars Sancum voce canebant, "Ils avançaient, et, dans leur allégresse, les uns chantaient Sancus ${ }^{35}$. On note ici le rapprochement sémantique et métrique que l'on peut faire entre l'adjectif sancti de Flodoard et l'adjectif laeti de Silius Italicus. Qui plus est, le sentiment de joie est aussi présent chez l'auteur médiéval avec le participe gaudentes. Ce vers constitue donc une réécriture de Silius Italicus, qui, loin de faire perdre de vue la dimension chrétienne du poème (sancti prend la place de laeti), lui apporte une forte dimension épique. Enfin, pour clore l'analyse du passage, arrêtons-nous encore sur le vers 814. La fin (-que potentem) en est tout à fait classique. On la trouve notamment chez Virgile (A. 1,$80 ; 2$, $296 ; 3,438 ; 6,247 ; 6,621 ; 6,843 ; 7,234)$, Ovide (Met. 6, 426), Lucain $(1,2 ; \mathrm{I}, 83 ; 8,762$; 9, 1002) et Stace (Silu. 5, 1, 187; 5, 2, 29; Theb. X11, 270). Mais il est intéressant de s'arrêter sur la retractatio virgilienne de l'Énéide 6, 247 : uoce uocans Hecaten caeloque Ereboque potentem, "appelant à haute voix Hécate puissante au ciel et dans l'Érèbe » (il s'agit du passage où la Sibylle invoque Hécate). En effet, on trouve précisément en début de vers chez Flodoard le verbe inuocat. L'hypotexte de Prudence et les réminiscences païennes se complètent.

Le fait que Flodoard ne subordonne pas les réminiscences au texte de Prudence apparaît au vers 772: Vt stent exhortans contra dicantque tyranno. La place du nom tyrannus en fin de vers est tout à fait classique dans l'épopée. Mais la mention contra dicantque fait explicitement référence à Virgile (A. 10, 448 : Talibus et dictis it contra dicta tyranni). En pleine scène de combat, Pallas prend la parole pour répondre au tyran Turnus. Chez Flodoard, l'expression empruntée à Virgile ne se situe pas à la même place dans le vers. Il s'agit donc encore une fois d'une retractatio qui n'est pas uniquement formelle. Mais la retractatio est double dans ce vers 772 doté d'une coupe penthémimère qui le divise symboliquement avec, d'un côté, l'expression contra dicantque tyranno qui est une référence virgilienne et, de l'autre, l'expression ut stent exhortans qui constitue une réécriture de Prudence. On lit en effet aux vers 54-55 de Peristephanon 10 : animos pauentum praestruens hortatibus / stent ut parati neue cedant turbini, « [Romain] fortifie de ses exhortations les âmes des fidèles apeurés, pour qu'ils se tiennent prêts et ne plient pas devant la tempête ». Nous notons en gras les termes repris directement à Prudence. Le vers de Flodoard est donc symboliquement partagé entre sources chrétiennes et sources païennes mais la conjonction de coordination -que illustre la fusion qu'il opère entre les deux.

Pour conclure, si l'on en revient aux vers d'ouverture du chapitre consacré à Romain chez Flodoard, on peut lire différemment la proposition du vers 765 , quare satis est libare 
tropaea. Flodoard annonce ainsi qu'il ne développera pas le récit du martyre de Romain, mais se contentera de consacrer, de faire une libation en l'honneur de ce saint. D'ailleurs, un autre sens du verbe libare peut être "toucher » au sens d'« effleurer un sujet ", ce qui suggère la breuitas. Mais, nous l'avons vu, son poème est loin d'être aussi simple qu'il ne le laisse entendre. Peut-être faut-il dans ce cas lire libare au sens de "prendre un passage d'un auteur", tel qu'on le trouve employé chez Ambroise de Milan ${ }^{36}$. Cela souligne bien le lien, le dialogue que Flodoard établit entre divers auteurs. Ce dialogue est mis en place dès le vers 767 : Ecclesiamque dolo meditans irrumpere furuo. Le verbe irrumpere se trouve déjà chez Prudence (templum cogitans inrumpere, v. 46). L'utilisation du même verbe et le parallèle entre les participes cogitans et meditans montrent explicitement le fait que Flodoard revendique sa source. Toutefois, il n'emploie pas le nom templum mais le nom ecclesiam. Or, c'est ce nom que l'on lit dans le Martyrologe d'Adon (18, XI : ecclesiam irrumpere). Et l'on sait qu'Adon constitue l'une des sources principales de Flodoard ${ }^{37}$. Celui-ci procède donc à une première fusion entre des auteurs chrétiens. Mais le fait d'utiliser le verbe irrumpere à cette place métrique introduit une résonnance avec les auteurs latins païens puisqu'on le retrouve au même endroit chez Lucain $(5,127$ : corripuit cogitque fores irrumpere templi) et Silius Italicus (5, 183 : Cinyphiae que globos dubitarim irrumpere turmae ; $10,367:$... nec enim sacris irrumpere muris ; 13, 79: haec ausos Celtas irrumpere moenia Romae ; 13, 175 : confisum nullo comitante irrumpere tectis ; 14, 580 : nec mora quin trepidos hac clade irrumpere muros). La référence au livre 13 de la Guerre punique est particulièrement intéressante car il est question de l'invasion des remparts de Rome. Et chez Lucain, il s'agit de faire irruption dans les portes du temple. Par l'intertextualité, Flodoard établit un pont littéraire entre des auteurs, dont les principaux pour la littérature païenne sont Virgile, Ovide, Lucain et Silius Italicus, et donne une dimension épique très forte au combat de Romain. Il ne s'agit pas pour Flodoard de subordonner la littérature épique aux valeurs chrétiennes, mais de permettre un éclairage réciproque, une sorte de fusion par laquelle la littérature païenne rehausse par sa dimension épique les victoires et les valeurs chrétiennes tandis que ces dernières permettent un renouveau de l'épopée.

\section{Auteurs antiques et médiévaux}

Bibliotheca hagiographica latina antiquae et mediae aetatis, Bruxelles, Société des Bollandistes, 1898-1901, 2 vol.

Le martyrologe d'Adon: ses deux familles, ses trois recensions. Texte et commentaire, éd. J. DUBOIS, G. RENAUD, Sources d'histoire médiévale 16, Paris, Éd. du Centre national de la recherche scientifique, 1984.

ARISTOTE, Poétique, texte établi et trad. par J. HARDY, CUF, Paris, Les Belles Lettres, 1932. 
Flodoardi, canonici remensis. Opera omnia..., éd. J.-P. MIGNE, Patrologiae Cursus Completus. Series Secunda 135, Petit-Montrouge, J.-P. Migne, 1853.

LUCAIN, La guerre civile : la Pharsale. I, Livres I-V, texte établi et trad. par A. BOURGERY, CUF, Paris, Les Belles Lettres, 1947 (2 éd., rev. et corr. : 1997).

LUCAIN, La guerre civile : la Pharsale. II, Livres VI-X, texte établi et trad. par A. BOURGERY, M. PONCHONT, CUF, Paris, Les Belles Lettres, 1947 (6 $6^{\mathrm{e}}$ tirage revu et corr. : 1993).

LUCRÈCE, De la Nature. I, Livres I-IV, texte établi par A. ERNOUT, CUF, Paris, Les Belles Lettres, 1920.

LUCRÈCE, De rerum natura. III, Livres V et VI, par A. ERNOUT et L. ROBIN, $2^{\mathrm{e}}$ éd., Coll. de commentaires d'auteurs anciens, Paris, Les Belles Lettres, 1962.

ovIDE, Les Métamorphoses. I, Livres I-V, texte établi et trad. par G. LAFAYE, $8^{\mathrm{e}}$ éd. revue et corr., CUF, Paris, Les Belles Lettres, 1994.

OVIDE, Les Métamorphoses. II, Livres VI-X, texte établi et trad. par G. LAFAYE, CUF, Paris, Les Belles Lettres, 1955 ( $6^{\mathrm{e}}$ tirage revu et corr. : 1989).

OVIDE, Les Métamorphoses. III, Livres XI-XV, texte établi et trad. par G. LAFAYE, $3^{\mathrm{e}}$ tirage rev. et corr. par H. Le Bonniec, CUF, Paris, Les Belles Lettres, 2002.

PRUDENCE, Le livre des Couronnes, texte établi et trad. par M. LAVARENNE, CUF, Paris, Les Belles Lettres, 1963.

SILIUS ITALICUS, La Guerre punique. I, Livres I-IV, texte établi et trad. par P. MINICONI et G. DEVALLET, CUF, Paris, Les Belles Lettres, 1979.

SILIUS ITALICUS, La Guerre punique. II, Livres V-VIII, texte établi et trad. par J. VolPILHAC, P. MINICONI et G. DEVALLET, CUF, Paris, Les Belles Lettres, 1981 (2 $2^{\mathrm{e}}$ tirage : 2003).

SILIUS ITALICUS, La Guerre punique. III, Livres IX-XIII, texte établi et trad. par J. VOLPILHAC-LENTHÉRIC, M. MARTIN, P. Miniconi et G. DEVALLET, CUF, Paris, Les Belles Lettres, 1984.

SILIUS ITALICUS, La Guerre Punique. IV, Livres XIV-XVII, texte établi et trad. par M. MARTIN et G. DEVALLET, CUF, Paris, Les Belles Lettres, 1992.

STACE, Thébaïde. I, Livres I-IV, texte établi et trad. par R. LESUEUR, CUF, Paris, Les Belles Lettres, 1990.

STACE, Thébaïde. II, Livres V-VIII, texte établi et trad. par R. LESUEUR, CUF, Paris, Les Belles Lettres, 1991.

STACE, Thébaïde. III, Livres IX-XII, texte établi et trad. par R. LESUEUR, CUF, Paris, Les Belles Lettres, 1994.

STACE, Silves. I, Livres I-III, texte établi par H. FRÈRE et trad. par H. J. IZAAC, $3^{\mathrm{e}}$ tirage revu et corr., CUF, Paris, Les Belles Lettres, 1992.

STACE, Silves. II, Livres IV-V, texte établi par H. FRÈRE et trad. par H. J. IZAAC, $2^{\mathrm{e}}$ éd. revue et corr., CUF, Paris, Les Belles Lettres, 1961.

VIRGILE, Énéide. I, Livres I-IV, texte établi et trad. par J. PERRET, $4^{e}$ tirage rev. et corr. par R. LESUEUR, CUF, Paris, Les Belles Lettres, 1995.

VIRGILE, Énéide. II, Livres V-VIII, texte établi et trad. par J. PERRET, CUF, Paris, Les Belles Lettres, 1978. 
VIRGILE, Énéide. III, Livres IX-XII, texte établi et trad. par J. PERRET, $2^{\mathrm{e}}$ tirage rev. et corr., CUF, Paris, Les Belles Lettres, 1987.

\section{Études modernes}

CHARLET J.-L. 1980, «L'apport de la poésie latine chrétienne à la mutation de l'épopée antique : Prudence précurseur de l'épopée médiévale », BAGB 2, p. 207-217.

- 1995, «L'épopée latine aux IV ème et vè̀me siècles », in G. REGGI (a cura di), Aspetti della poesia epica latina. Atti del corso d'aggiornamento per docenti di latino e greco del Canton Ticino, Lugano 21-22-23 ottobre 1993, Lugano, G. Casagrande - Ed. universitarie della Svizzera italiana, p. 207-225. DELEHAYE H. 1933, Les origines du culte des martyrs, $2^{\mathrm{e}}$ éd. revue, Subsidia hagiographica 20 , Bruxelles, Société des Bollandistes.

DEPROOST P.-A. 1997, « L'épopée biblique en langue latine. Essai de définition d'un genre littéraire », Latomus 56, p. 14-39.

DOLBEAU F. 2002, « Un domaine négligé de la littérature médiolatine : les textes hagiographiques en vers », Cahiers de civilisation médiévale 45, p. 129-139.

FONTAINE J. 1976, « Hagiographie et politique de Sulpice Sévère à Venance Fortunat », Revue d'histoire de l'Église de France 62, p. 113-140.

FONTAINE J., PERRET J. 1981, Naissance de la poésie dans l'Occident chrétien. Esquisse d'une histoire de la poésie latine chrétienne $\mathrm{du} \mathrm{III}^{e}$ au VI $\mathrm{I}^{e}$ siècle, Coll. des études augustiniennes. Série Antiquité 85, Paris, Études augustiniennes.

FUX P.-Y. 2003, Les Sept passions de Prudence (Peristephanon 2.5.9 11-14). Introduction générale et commentaire, Paradosis 46, Fribourg, Éditions universitaires.

GAIFFIER D'HESTROY B. de 1947, «L'hagiographe et son public au XI ${ }^{\mathrm{e}}$ siècle », in Miscellanea historica in honorem Leonis van der Essen. Universitatis Catholicae in oppido Lovaniensi iam annos XXXV professoris, Paris - Bruxelles, Éditions universitaires, p. 135-166.

- 1970, « Hagiographie et historiographie », in La Storiografia Altomedievale. [17a] Settimana di studio del Centro italiano di studi sull'Alto Medioevo, [Spoleto], 10-16 aprile 1969, Settimane di studio del Centro Italiano di studi sull'Alto Medioevo 17, Spolète, Centro italiano di studi sull'Alto Medioevo, p. 139-166.

GOSSEREZ L. 2009, «Théâtralité du Peristephanon de Prudence », in D. VAN MAL-MAEDER, A. BURNIER, L. NÚÑEZ (éd.), Jeux de voix. Énonciation, intertextualité et intentionnalité dans la littérature antique, Echo 8, Berne, P. Lang, p. 347-367.

GOULLET M. 2001, « La laudatio sanctorum dans le haut Moyen Âge, entre uita et éloge », in L. MARY, M. sот (éd.), Le discours d'éloge entre Antiquité et Moyen Âge, Paris, Picard, p. 141-152.

-2003, « Une typologie des réécritures peut-elle éclairer la nature du discours hagiographique?», Hagiographica 10, p. 109-121.

- 2005, Écriture et réécriture hagiographiques. Essai sur les réécritures de Vies de saints dans l'Occident latin médiéval (VIII -XIII siècle), Hagiologia 4, Turnhout, Brepols.

- 2008, « Poésie hagiographique et didactique de la poésie », in P. STOTz (Hrsg.), Dichten als StoffVermittlung. Formen, Ziele, Wirkungen. Beiträge zur Praxis der Versifikation lateinischer Texte im Mittelalter, Medienwandel, Medienwechsel, Medienwissen 5, Zürich, Chronos, p. 71-84. 
GOULLET M., HEINZELMANN M. (dir.) 2003, La réécriture hagiographique dans l'Occident médiéval. Transformations formelles et idéologiques, Beihefte der Francia 58, Ostfildern, J. Thorbecke.

INGLEBERT H. 1994, « Les héros romains, les martyrs et les ascètes : les uirtutes et les préférences politiques chez les auteurs chrétiens latins du $\mathrm{III}^{\mathrm{e}}$ au v $\mathrm{v}^{\mathrm{e}}$ siècle », REAug 40/2, p. 305-325.

ISAÏA M.-C., GRANIER T. (éd.) 2014, Normes et hagiographie dans l'Occident latin (VI ${ }^{e}-X V I^{e}$ siècles). Actes du colloque international de Lyon, 4-6 octobre 2010, Hagiologia 9, Turnhout, Brepols.

JACOBSEN P.C. 1978, Flodoard von Reims. Sein Leben und seine Dichtung 'De triumphis Christi', Mittellateinische Studien und Texte 10, Leyde, Brill.

LABARRE S. 2010, «Écriture épique et édification religieuse dans l'hagiographie poétique $\left(\mathrm{V}^{\mathrm{e}}-\mathrm{VI}^{\mathrm{e}} \mathrm{s}.\right)$ : les scènes de résurrections ", Rursus. Poiétique, réception et réécriture des textes antiques 5 [en ligne]. URL : https://rursus.revues.org/408.

NOUGARET L. 1986, Traité de métrique latine, $4^{\mathrm{e}}$ éd. corrigée, Nouvelle coll. à l'usage des classes. Première série 36, Paris, Klincksieck.

PETIT P. 1978, Histoire générale de l'Empire romain. III, Le Bas-Empire (284-395), Paris, Éd. du Seuil.

PHILIPPART G. 1998, «L'hagiographie comme littérature », Revue des sciences humaines 251, p. 11-39.

Sот M. 1990, « L'expérience visionnaire et sa fonction dans l'Histoire de l'église de Reims de Flodoard », in M. sот (dir.), Haut Moyen Âge. Culture, éducation et société. Études offertes à Pierre Riché, Nanterre, Éd. Publidix - La Garenne-Colombes, Éd. européennes Érasme, p. 477-491.

-1993, Un historien et son Église au $X^{e}$ siècle : Flodoard de Reims, Paris, Fayard.

\section{NOTES}

1. Texte établi par nos soins. Le texte de Flodard que nous citerons a été établi à partir des deux manuscrits contenant le De Triumphis Christi Antiochiae gestis, un manuscrit du $\mathrm{x}^{\mathrm{e}} \mathrm{s}$. (Paris, Bibliothèque Sainte-Geneviève, 2409) et un manuscrit du xvII ${ }^{\mathrm{e}} \mathrm{s}$. (Paris, Bibliothèque Mazarine, 3866).

2. Dioclétien revendique sa fidélité à la religion polythéiste romaine. Mais le début de son règne (de 284 à 303) est marqué par une certaine tolérance à l'égard des chrétiens. C'est en 303 que débute une des plus virulentes persécutions à l'égard des chrétiens. À ce sujet, voir PETIT 1978, p. 37-41.

3. Si l'on met de côté la mention de Luc $(1,3$, v. 43): Quam meminit Lucas. Mais nous isolons cette référence comme particulière car il s'agit d'un renvoi au Nouveau Testament.

4. Pour l'œuvre de Prudence, nous citerons tout au long de cet article le texte et la traduction établis par M. Lavarenne dans la CUF (1963).

5. GOULET 2005, p. 23.

6. En ce qui concerne la recherche des sources de Flodoard pour le De Triumphis Christi, nous renvoyons à JACOBSEN 1978, ouvrage très complet, et en particulier aux p. 240-243 qui recensent les sources pour chaque chapitre de la partie sur Antioche.

7. Nous comptons parmi eux la Praefatio qui comprend 40 vers.

8. GOULLET 2005, p. 38. 
9. GOULLET 2005, p. 38.

10. M. Goullet explique ainsi qu'un « corollaire de la réécriture en meilleur style » est "celui de la réécriture en style plus simple», ce qui passe notamment par la breuitas (GOULLET 2005).

11. Bibliotheca hagiographica latina antiquae et mediae aetatis, Bruxelles, Société des Bollandistes, 1898-1901, 2 vol.

12. Par "chevilles", nous entendons des mots ou des formules qui servent à combler des manques dans le vers et qui peuvent s'adapter tels quels sur certains pieds.

13. NOUGARET 1986, p. 60. On trouve déjà cette idée chez Aristote, Po.

14. GOSSEREZ 2009.

15. Aristote, Po. 13, 1452 b 28 - 15, 1454 b 18.

16. FUX 2003, «Introduction », p. 52.

17. FUX 2003, «Introduction », p. 52.

18. LABARRE 2010.

19. Pour les Métamorphoses d'Ovide, nous citons le texte et la traduction établis par G. Lafaye dans la CUF.

20. DEPROOST 1997, p. 30.

21. LABARRE 2010 , p. 3.

22. FUX 2003, p. 15.

23. FUX 2003, n. 112 p. 20.

24. Pour l'Énéide de Virgile, nous citons le texte et la traduction établis par J. Perret dans la CUF (4 tirage de l'éd. revue et corr., 1995).

25. Nous soulignons.

26. Pour le Bellum Ciuile de Lucain, nous citons le texte et la traduction établis par A. Bourgery dans la CUF (1947).

27. DEPROOST 1997, p. 21.

28. DEPROOST 1997, p. 24.

29. Le nom athleta pour désigner les martyrs est employé par Flodoard à plusieurs reprises $(1,125 ; 1,485 ; 1,567 ; 1,645 ; 1,722 ; 2,35)$.

30. DOLBEAU 2002, p. 136.

31. On le trouve en particulier chez Virgile $(A .5,359 ; 12,397)$ ou Ovide (Met. 1, $524 ; 2$, $618 ; 6,152 ; 7,138 ; 7,562 ; 8,188 ; 8,215 ; 8,866 ; 9,62 ; 9,66 ; 15,484)$.

32. DEPROOST 1997, p. 31.

33. Sот 1990, р. 489.

34. Virgile (A. 3, $585 ; 12,365)$; Ovide (Met. 5, 286) ; Lucain (10, 242) ; Silius Italicus (1, $535 ; 5,34$ où l'on observe nubila caelum) ; Stace (Silu. 3, 3, 36; Theb. 1, 342; 1, 664; 6, 406).

35. Pour la Guerre punique de Silius Italicus, nous citons le texte et la traduction établis par P. Miniconi et G. Devallet dans la CUF. 
36. Dans son Dictionnaire Latin-Français des auteurs chrétiens, A. Blaise cite comme exemple pour cet emploi du verbe libare Ambroise de Milan (Expositio euangelii secundum Lucam, CSEL 32, IV) : Haec de Matthaeo pauca libauimus.

37. JACOBSEN 1978, p. 240-243.

\section{RÉSUMÉS}

Cette étude a pour objet le chapitre 14 du livre 1 du De Triumphis Christi Antiochiae gestis de Flodoard à travers une traduction inédite. Consacré à saint Romain, il constitue le seul récit de martyre du De Triumphis Christi Antiochiae gestis qui se présente explicitement comme une réécriture, en l'occurrence du Peristephanon 10 de Prudence qui ne compte pas moins de 1140 vers résumés en 81 vers par Flodoard. Sous couvert de recourir à la breuitas, il transpose le texte de Prudence, qui écrit en mètre iambique, en hexamètres dactyliques. La retractatio de Flodoard efface le caractère théâtral du poème de Prudence pour accentuer la dimension épique du martyre de Romain. En faisant dialoguer les réminiscences du Peristephanon avec des réminiscences de la poésie épique païenne, il glorifie les victoires chrétiennes tandis que ces dernières permettent un renouveau de l'épopée.

This article is based on a first ever translation of Flodoard's De Triumphis Christi Antiochiae gestis, Book I, Chapter XIV. The chapter, narrating St. Romanus' martyrdom, is the only one of that kind in Flodoard's poem. It is an explicit 81 verses summed up retractatio by Flodoard of the 1.140 verses constituting Prudentius' Peristephanon X. Gaving breuitas as a pretext, he transposes Prudentius' iambic meters in dactylic hexameters. In order to lay emphasis on the epic dimension of Romanus' martyrdom, Flodard's retractatio erases the theatrical dimension of Pudentius' lines. As a way to both glorify Christian victories and to renew epic poetry, he puts reminiscences of Peristephanon in dialogue with reminiscences of pagan epic poetry.

\section{INDEX}

Mots-clés : réécriture, retractatio, poésie épique

Index géographique : Antioche

Keywords : retractatio, latin epic, epic poetry

nomsmotscles Adon, Ambroise, Aristote, Cassiodore, Catulle, Eusèbe de Césarée, Flodoard de Reims, Grégoire de Tours, Horace, Jérôme, Juvénal, Lucain, Lucrèce, Ovide, Properce, Prudence, Rufin d'Aquilée, Silius Italicus, Sénèque, Stace, Virgile

\section{AUTEURS}

\section{JULIE LEYRONAS}

Université Jean Moulin Lyon 3, UMR 5189 HiSoMA 